\title{
Effect of Glycosylation and Glucose Trimming Inhibitors on the Influenza A Virus Glycoproteins
}

\author{
Takehiko SAITO* and Isao YAMAGUCHI \\ Department of Animal Science, Faculty of Agriculture, Kobe University, 1-1 Rokkodai-cho, Nada-ku, Kobe 657-8501, Japan
}

(Received 24 November 1999/Accepted 2 February 2000)

ABSTRACT. N-glycosylation and glucose trimming of the influenza virus hemagglutinin (HA) and neuraminidase (NA) were studied by using glycosylation inhibitor (tunicamycin; TM) and glucosidase inhibitors. TM treatment of MDCK cells infected with a reassortant virus NWS-N8 resulted in reduced transport of the viral glycoproteins to the cell surface. The degree of the effects differed between the HA and the NA ( $80 \%$ reduction for the HA and 97\% reduction for the NA), indicating a difference in dependency on N-glycosylation between these glycoproteins. Differential dependency on glucose trimming was clearly demonstrated when the surface transport of the glycoproteins was compared after treatment of the virus-infected cells with glucosidase inhibitors. Fluorescence-activated cell sorting (FACS) analysis revealed that the surface transport of the NA reduced to 50\% after castanospermine (CST) treatment but not did that of the HA. An anti-viral effect of a glucosidase inhibitor on the NWS-N8 strain was also demonstrated. The correlation between the expression of the NA on the cell surface and virus yield suggests that CST may interfere with virus release through its effect on the NA.-KEY WORDS: glucosidase inhibitor, glycoprotein, glycosylation, influenza.

J. Vet. Med. Sci. 62(6): 575-581, 2000

Influenza A virus has two membrane-bound glycoproteins, the hemagglutinin (HA) and the neuraminidase (NA) [14]. The HA is the type I glycoprotein, binding to a sialic acid-containing receptor on the host cell surface. It also mediates the fusion process of the virus particle with the endosomal membrane. The NA is the type II glycoprotein, serving as a receptor-destroying enzyme. It catalyzes cleavage of the $\alpha$-ketosidic linkage between a terminal sialic acid residue and the adjacent sugar residue.

$\mathrm{N}$-linked oligosaccharides on glycoproteins undergo the sequential trimming process during the transport pathway to their final destination [9]. The core unit of oligosaccharide is transferred to the asparagine side chain at the glycosylation consensus sequence (Asn-X-Ser/Thr) from a dolichol pyrophosphate precursor. It is then subjected to the first step of trimming in the endoplasmic reticulum (ER) by glucosidase I. It removes the $\alpha-1,2-$ linked glucose from the three terminal glucose residues. Following this, glucosidase II catalyses the removal of the two remaining $\alpha-1,3$-linked glucoses. After $\alpha-1,2$ mannosidase removes two external mannose residues from the core, the glycoprotein is transported to the Golgi apparatus for further trimming.

Recent evidence suggests that trimming of $\mathrm{N}$-linked oligosaccharides in the ER plays an important role in glycoprotein maturation $[5,7,12,15,22]$. Maturation of glycoproteins often depends upon the extent of trimming of $N$-linked oligosaccharide side chains, for inhibitors of glucosidases I and II such as castanospermine (CST), 1deoxynojirimycin (dNM) and bromoconduritol (BCT)

* Present address: Saito, T., National Institute of Infectious Diseases, 1-23-1 Toyama, Shinjuku-ku, Tokyo 162-8640, Japan. impair the folding of some glycoproteins. The ER molecular chaperone calnexin is suggested to bind to the monoglucosylated $N$-linked oligosaccharides which are present only on incompletely folded glycoproteins $[5,7$, 22]. Impaired folding of glycoproteins caused by glucosidase inhibitors appears to account for the failure of glycoproteins to bind calnexin or the other lectin-like chaperones.

Recently, Hebert et al. [8] presented evidence of the involvement of calnexin and calreticulin in correct folding and oligomerization of in vitro translated influenza HA using canine pancreas microsomes. Yet, dependence of glycoprotein maturation on glycosylation and trimming of oligosaccharides differs among glycoproteins, indicating differential involvement of lectin-like chaperones in proper folding and oligomerization of glycoproteins.

In this study, we compared the surface expression of the $\mathrm{HA}$ and the NA of reassortant virus NWS-N8 on the host cell surface, in the presence of tunicamycin (TM) or glucosidase inhibitors. The results indicate a differential effect of carbohydrate processing on viral glycoprotein transport, demonstrating that the HA is less dependent on the carbohydrate moiety for its maturation and that the transport of the NA to the cell surface is made less efficient by treatment with a glucosidase inhibitor.

\section{MATERIALS AND METHODS}

Virus, Monoclonal antibodies (MAbs) and Cells: Reassortant virus NWS-duck/Ukraine/1/63 (H1N8: NWSN8) was used [18]. The virus has the HA of A/NWS/33 (H1N1) and the NA of A/duck/Ukraine/1/63 (H3N8). MAbs raised against the NA of A/duck/Ukraine/1/63 were reported elsewhere [18]. The MAb against WSN HA, which is cross-reactive with NWS HA, was kindly provided 
by Dr. R. G. Webster, St. Jude Children's Research Hospital. Madin-Darby canine kidney (MDCK) cells were maintained in Eagle's minimal essential medium (MEM), supplemented with 5\% newborn calf serum.

Indirect immunofluorescence (IF) analysis: Cells were grown overnight on acid-cleaned cover slips placed in a 6well culture plate, then infected with virus in $600 \mu \mathrm{l}$ of MEM supplemented with $0.3 \%$ bovine serum albumin (BSA) (MEM-BSA) at an m.o.i of 3. After a 1-hr infection period, the virus-containing medium was replaced with fresh MEM-BSA and the cells were incubated for additional $4.5 \mathrm{hr}$ with or without drug. After incubation, the cover slips were removed from the plate and washed with PBS three times. The cells were fixed with $3 \%$ freshly prepared paraformaldehyde in PBS for $10 \mathrm{~min}$ at $4{ }^{\circ} \mathrm{C}$. To make the cells permeable, fixed cells were treated with $0.5 \%$ Triton $\mathrm{X}-100$ in PBS for $20 \mathrm{~min}$ at $4{ }^{\circ} \mathrm{C}$. Immunoreactions were performed by incubation with 100 $\mu l$ of MAb diluted in $1.5 \%$ BSA-PBS at $4{ }^{\circ} \mathrm{C}$ overnight. After the cells were washed with PBS, they were incubated with anti-mouse IgG FITC conjugate (Cappel) for $30 \mathrm{~min}$ at room temperature, then washed with PBS three times. Inverted cover slips were mounted on a microscope slide with GEL/MOUNT (Biomeda) and observed under a microscope (Nikon OPTIPHOT).

Fluorescence-activated cell sorting (FACS) analysis: A confluent monolayer of MDCK cells was infected with NWS-N8 virus as described above. After 5-hr infection, the cells were suspended by trypsinization at $37^{\circ} \mathrm{C}$ [13]. The cells were centrifuged $(15,000 \times \mathrm{g})$ for $1 \mathrm{~min}$ and washed twice with PBS, then fixed with $3 \%$ paraformaldehyde in PBS at $4^{\circ} \mathrm{C}$ for $30 \mathrm{~min}$. After being washed twice with PBS, the cells were incubated with MAb N8-4 (1:200 dilution in PBS) at $4^{\circ} \mathrm{C}$ overnight. After they were washed twice with PBS, they were incubated with anti-mouse IgG FITC conjugate (Cappel) for $1 \mathrm{hr}$ at room temperature. Then they were washed and resuspended in 1\% NBCS-PBS for FACS analysis with EPICS Profile II (Coulter).

Neuraminidase assay: For NA assay with cell-associated NA, virus-infected cells were solubilized with cell lysis buffer (50 mM Tris- $\mathrm{HCl}, \mathrm{pH} 7.5 ; 100 \mathrm{mM} \mathrm{NaCl} ; 1 \%$ Triton $\mathrm{X}-100 ; 20 \mathrm{mM}$ iodoacetamide). Samples were subjected to neuraminidase assay by the method recommended by the World Health Organization [1].

Plaque assay: The plaque-forming unit of culture medium in the presence of trypsin $(5 \mu \mathrm{g} / \mathrm{m} l)$ was determined as described by Klenk et al. [10].

Chemicals: CST, dNM, BCT and TM were purchased from Sigma Chemical Co. Cells were treated with drug at the final concentrations of $1 \mathrm{mM}$ for glucosidase inhibitors and $10 \mu \mathrm{g} / \mathrm{m} l$ for TM. $N$-tosyl-L-lysine chloromethyl ketone (TLCK; Sigma Chemical Co.) was dissolved in 1 $\mathrm{mM} \mathrm{HCl}$ and added to the cell culture at the concentration of $50 \mathrm{mM}$. Vibrio cholerae NA, protease free, was purchased from Oxford Glyco Systems.

\section{RESULTS}

Differential dependency on $N$-linked glycosylation between the HA and the NA: Among several glycoproteins investigated, there appear to be considerable differences in how they depend upon N-linked glycosylation for their maturation and/or cellular transport. We first examined NWS-N8 virus-infected cells for expression of the HA and the NA in the presence of TM.

Surface expression of the HA and the NA of the virusinfected cells was measured by FACS in the presence of MAbs (Fig. 1). MAb N8-4 reacts only with the tetrameric $\mathrm{NA}$; therefore, it is possible to measure the amount of mature NA on the cell surface. On the other hand, MAb N8-10 reacts with both the monomeric and the oligomeric NAs and MAb N8-82 reacts with the oligomeric NAs [18, 19]. Oligomeric specificity of the MAb for the HA has not been determined. Surface expression of the HA in the presence of TM at $5 \mathrm{hr}$ p.i. decreased to $20 \%$ of that of the HA without TM treatment. On the other hand, surface expression of the NA in the presence of TM, stained with MAb N8-4, decreased to $3.1 \%$ of that of the NA in the absence of TM. A similar result was obtained when TMtreated virus-infected cells were stained with MAb N8-10 (data not shown). These results indicate that addition of $\mathrm{N}$ linked oligosaccharides is much more important for the cell surface transport of the NA molecule than that of the HA molecule.

Since the NA cannot oligomerize properly in the presence of TM [19], the reason for the impaired surface transport might be that the NA monomer without oligosaccharide side chains accumulated in the ER. To examine this possibility, IF analysis was carried out on the virus-infected cells. Cells were treated with TritonX-100 for intracellular staining (Figs. 2. b, c, e, f, h, and i). No intracellular accumulation of the NA by TM treatment was observed in the presence of MAb N8-10, N8-82 or N8-4. This suggests that the NA without oligosaccharide side chains may be degraded by cellular protease(s) and cannot accumulate enough for detection by IF analysis. TLCK treatment of the TM-treated virus-infected cells revealed a trace of intracellular staining of the NA. However, it did not greatly affect the cell surface expression of the NA (Fig. 1).

Taken together, these findings show that influenza virus NA of A/duck/Ukraine/63 origin is much more dependent on $\mathrm{N}$-linked oligosaccharides for its cell surface transport than the HA of NWS origin.

Effects of glucosidase inhibitors on glycoprotein maturation: $N$-linked oligosaccharide side chains not only provide solubility to polypeptide chains, but serve as the receptor for the ER chaperones such as calnexin and calreticulin, when it is in the mono-glucosylated form. It is believed that trimming of the oligosaccharide side chain is important for proper folding and quality control of glycoproteins. Because the TM treatment experiment above indicates that the NA appears more dependent on $\mathrm{N}$ - 


\section{$\alpha-H A$}

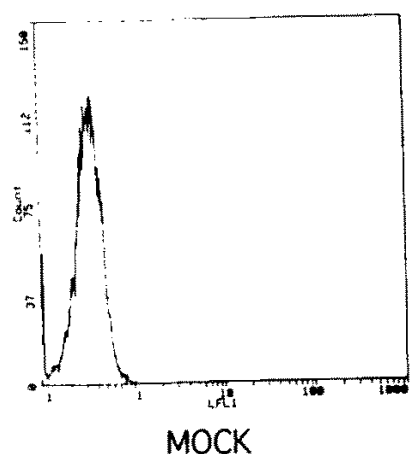

$\alpha$-NA (N8-4)

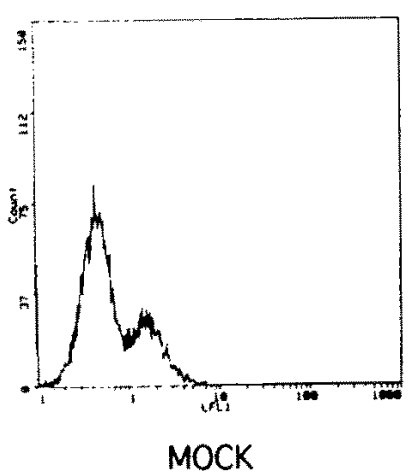

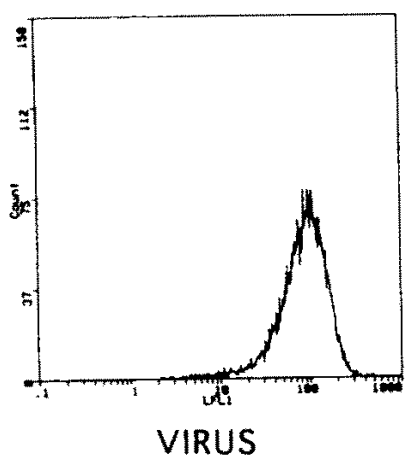

VIRUS

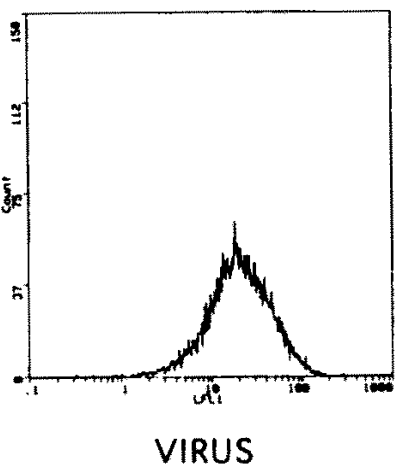

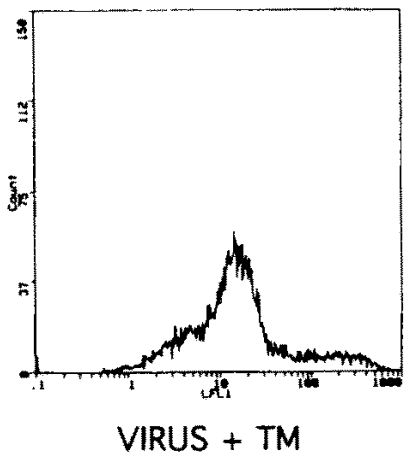
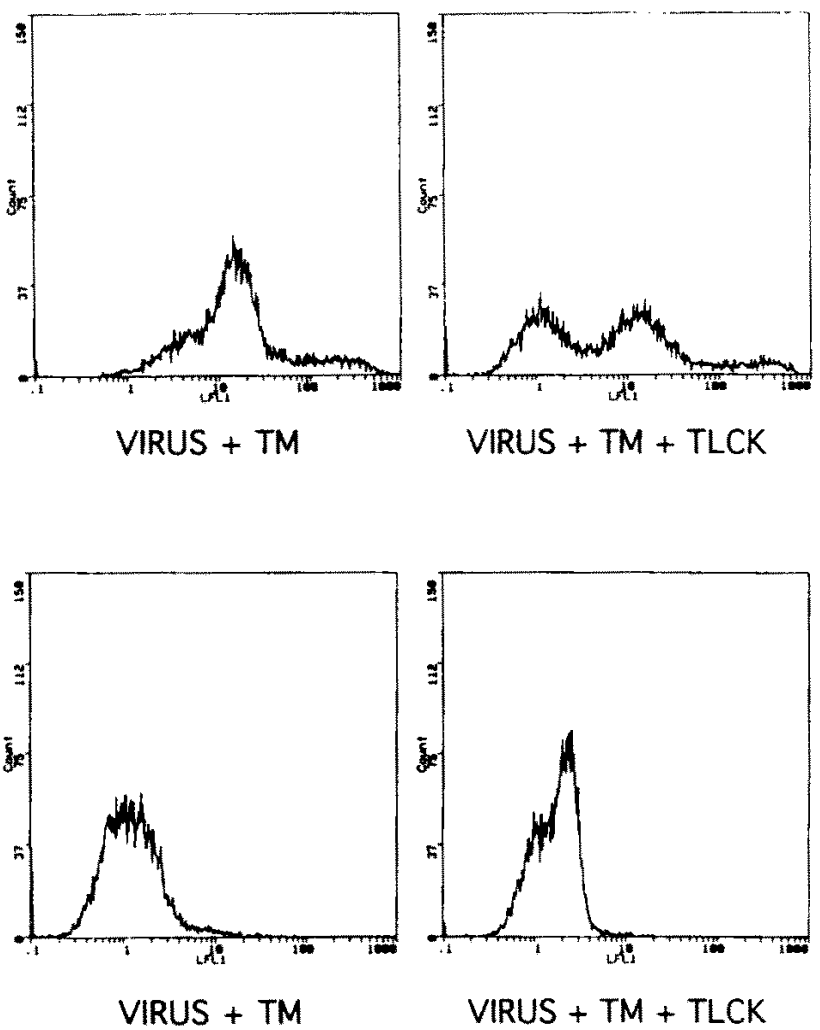

Fig. 1. Effect of TM on the amounts of HA and NA expressed on the surface of the virus-infected cells. Virus-infected cells were treated with TM or TM+TLCK for 5 hr p.i. The cells were then stained with anti-WSN HA MAb (top) or anti-N8 NA MAb N8-4 (bottom).

linked oligosaccharides for its transport, we next examined the effect of glucosidase inhibitors on the maturation and the transport of the N8 NA in the virus-infected cells.

Virus-infected cells were subjected to IF analysis with MAb N8-4 or N8-10 after 4.5-hr incubation with a glucosidase inhibitor. In CST-treated virus-infected cells, surface expression of the NA stained by MAb N8-4 was significantly lower than in the virus-infected cells without CST treatment (Figs. 3. a and b). Similarly, when dNMtreated cells were stained with MAb N8-4 or N8-10, decreased surface expression of the NA was observed (Figs. 4. $a, b, e$, and $f$ ). The amount of intracellular mature NA stained by MAb N8-4 was decreased in both CST- (Figs. 3. c and d) and dNM-treated (Fig. 4. g and h) cells. However, no significant difference was detected by IF analysis with MAb N8-10 between dNM-treated and untreated virusinfected cells by IF analysis (Figs. 4. $\mathrm{c}$ and d).

After obtaining the results of IF analysis, we quantitatively determined the surface expression of mature NA in CST-treated virus-infected cells by FACS analysis (Fig. 5). Surface expression of the NA was decreased to almost one-half in CST-treated virus-infected cells compared to virus-infected cells without treatment (mean fluorescence \pm S. D. with MAb N8-4; $8.2 \pm 4.3$ v.s. $15.3 \pm$
11.4). These observations, together with IF analysis, suggest that formation of the mature NA is impaired by glucosidase inhibitors. Consequently, a smaller amount of the mature NA reaches the host cell surface in cells treated with a glucosidase inhibitor.

For comparison, we also analyzed the surface expression of the HA in the same samples. No significant effect of CST on the HA expression was observed; the mean fluorescence \pm S. D. for CST-treated virus-infected cells was $125.5 \pm 65.7$ and $128.7 \pm 64.9$ for the virus-infected cells without treatment (Fig. 5). This clearly indicates that the importance of oligosaccharide trimming differs between the NA and the HA. Also, glucose trimming is not an absolute requirement for the maturation and cellular transport of these glycoproteins.

Anti-viral effect of glucosidase inhibitors on NWS-N8 strain: Since CST treatment resulted in $50 \%$ reduction of the surface expression of mature NA, there was a possibility that glucosidase inhibitors have an anti-viral effect on NWS-N8 strain. To explore this, HA titers, NA activity and PFU in the supernatants from glucosidase inhibitor-treated virus-infected cells were determined (Figs. 6. a and b).

All of the glucosidase inhibitors tested showed more than $50 \%$ reduction of the HA titer of the supernatants. No 


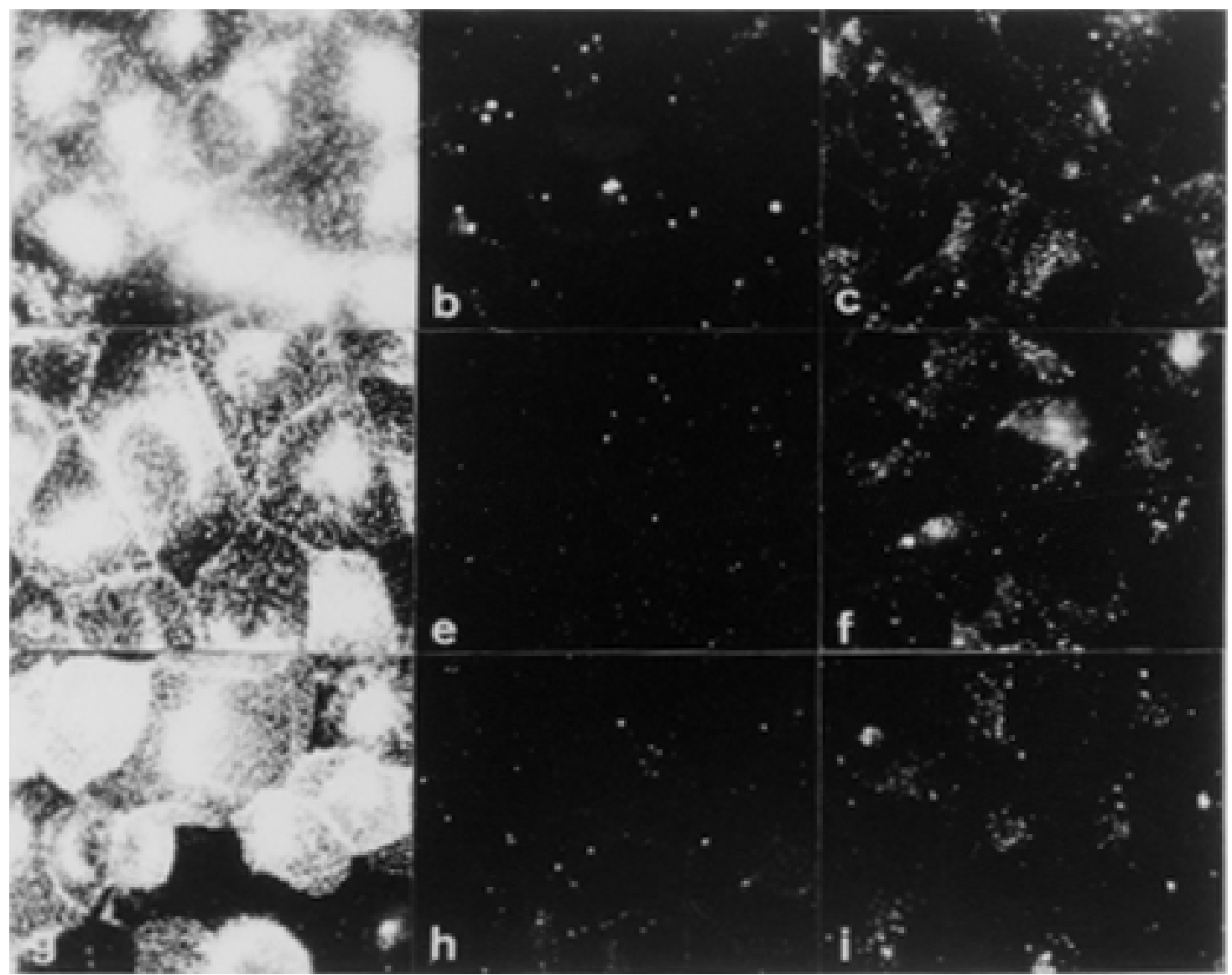

Fig. 2. Intracellular IF analysis of the TM-treated virus-infected cells. Virus-infected cells were treated with TM (b, e, and h) or TM + TLCK (c, f, and i) for $4.5 \mathrm{hr}$. Untreated cells (a, d, and g) were used as controls. Intracellular staining was carried out with MAb N8-10 (a, b, and c), N8-82 (d, e, and f) and N8-4 (g, h, and i).

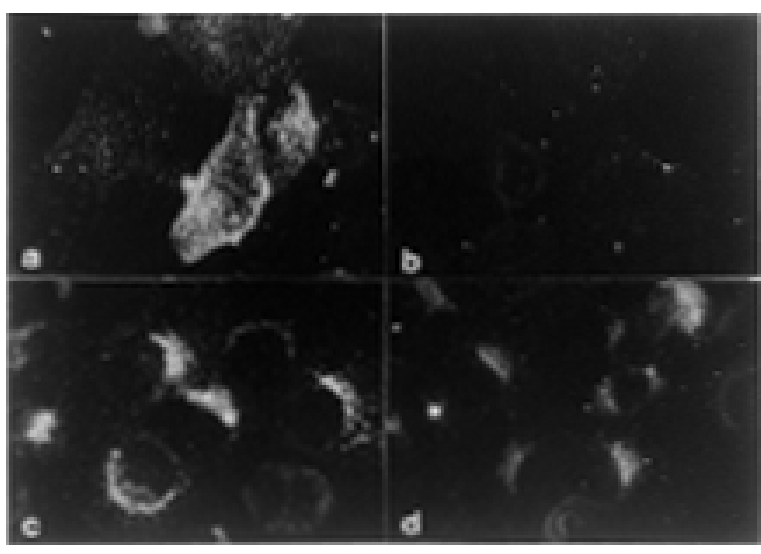

Fig. 3. Effect of CST treatment on the NA maturation. Virusinfected cells with (b and d) or without ( $a$ and c) CST treatment were examined at $4.5 \mathrm{hr}$ p.i. by IF analysis. Mature NAs were stained at the cell surface (a and b) or intracellulary (c and d) with MAb N8-4. significant difference in this effect was observed among the inhibitors tested. Also, NA activities of supernatants in the inhibitor-treated virus-infected cells were reduced to a range of about $30 \%$ to $60 \%$, depending on the inhibitors. $\mathrm{dNM}$ appears most effective among the inhibitors at the concentration examined. PFUs of supernatants of the inhibitor-treated virus-infected cells were decreased to about $30 \%$ of non-treated virus-infected cells. Again, no significant difference in the effect was observed among the three inhibitors.

These results clearly show an anti-viral effect of glucosidase inhibitors on NWS-N8 strain in vitro. Since CST treatment reduced the surface expression of the mature NA by $50 \%$, the amount of the mature NA protein at the cell surface may be the limiting factor for virus release from the inhibitor-treated virus-infected cells.

To determine if the reduction in the amount of enzymatic activity at the cell surface by CST treatment accounts for the reduction of virus particle release, exogenous NA was added to virus-infected cells along with CST. The exogenous NA did not affect the anti-viral activity of CST at two different concentrations $(5 \mathrm{mU} / \mathrm{m} l$ and $10 \mathrm{mU} / \mathrm{m} l)$, 


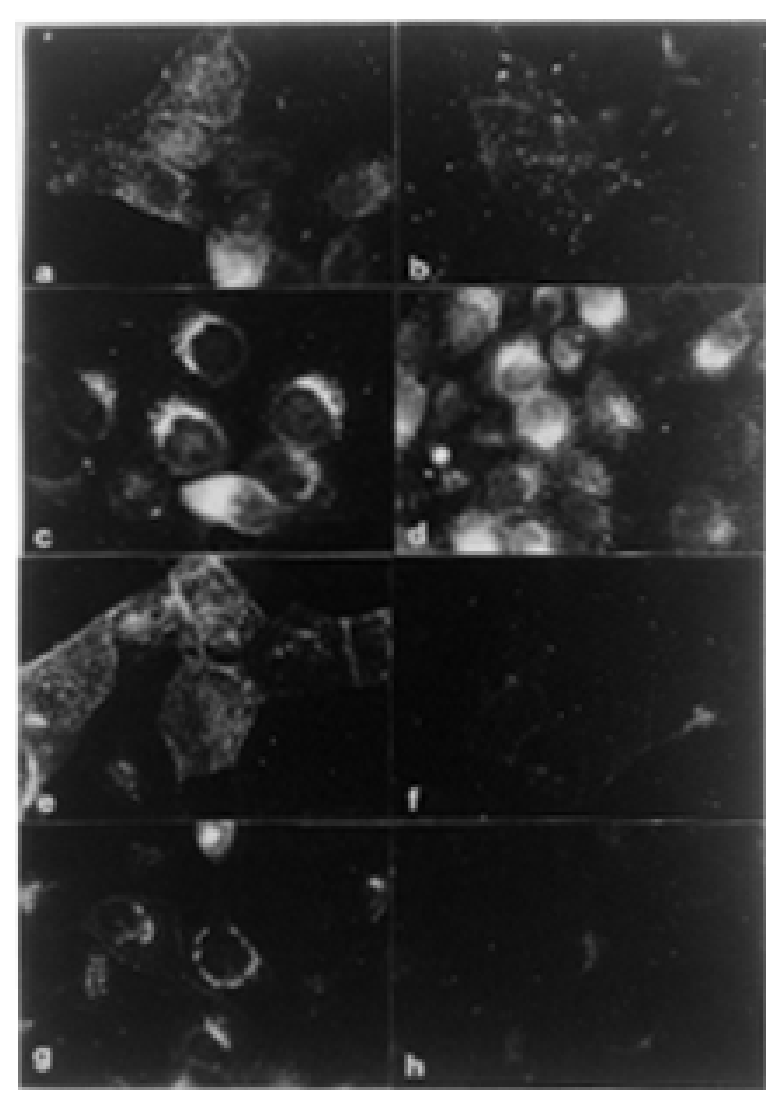

Fig. 4. Effect of dNM treatment on the NA maturation. Virusinfected cells with (b, d, f, and h) or without (a, c, e, and g) dNM treatment were examined at $4.5 \mathrm{hr}$ p.i. by IF analysis. NAs were stained at the cell surface (a, b, e, and $f$ ) or intracellulary (c, d, g, and h) with MAb N8-10 (a, b, c, and d) or N8-4 (e, f, g, and h).

indicating that the reduced amount of enzymatic activity may not be the limiting factor for the virus release (Table $1)$.

\section{DISCUSSION}

Glycoproteins generally depend upon sugar side chains for their folding and oligomerization, although the degree of the dependency varies among proteins. In this study, we showed that inhibition of $N$-linked glycosylation impairs cell surface transport of the NA. On the other hand, transport of the HA to the cell surface was not inhibited to the extent observed for the NA, indicating differential dependency on sugar chains between the HA and the NA. This differential dependency on sugar chains was clearer when the effect of CST on the cell surface transport of these proteins was examined. We found that, even in the presence of CST, the HA of NWS-N8 strain was transported as efficiently as in the absence of CST. This observation is consistent with a previous study [6]. It suggested that proper maturation and/or cellular transport of this glycoprotein does not depend greatly on the $N$-linked sugar chain modification governed by glucosidases. In contrast, cell surface transport of N8 NA was reduced by CST treatment to $50 \%$ compared with untreated virusinfected cells, showing that the NA depends more on the sugar chain modification for its maturation and/or cellular transport.

It has been suggested that the HA binds the molecular chaperone calnexin through its monoglucosylated form and that calnexin plays an important role in glycoprotein folding and quality control $[6,7]$. Calnexin binds to incompletely folded glycoproteins via partially glucosetrimmed oligosaccharides, facilitating their maturation in the ER $[2,6,8]$. CST inhibits glucose-trimming of $N$ linked oligosaccharides on glycoproteins catalyzed by the ER glucosidases, leaving the oligosaccharide chains in the triglucosylated form [16]. Although not yet demonstrated, the impaired transport of the NA by glucosidase inhibitors suggests that maturation of this glycoprotein may also be controlled by a calnexin-like chaperone. The effect of glucosidase inhibitors on the NA maturation may be the consequence of impaired binding to calnexin as suggested for other glycoproteins [8]. Yet, the mechanism of impaired transport of the NA by glucosidase inhibitors, along with whether the NA indeed interacts with calnexin, requires further study.

Glucosidase inhibitors are potent anti-viral reagents that are expected to be clinically useful against some viral infections such as those caused by Sindbis virus [4, 12], influenza virus $[3,4,17]$ and human immunodeficiency virus (HIV) [21], and others. CST, dNM and BCT show little effect on the cellular protein synthesis $[3,16,20]$ and we also did not observe any general cytotoxic effect of the glucosidase treatment at the concentration used in this study (data not shown). BCT inhibited virus particle release from fowl plague virus-infected chicken embryo cells, in contrast, no inhibition was observed in PR-8 infected cells $[3,4]$. It was suggested that the decreased virus particle release of fowl plaque virus from BCT-treated chicken embryo cells may be due to reduced stability of the HA caused by BCT treatment. Pan et al. [16] reported that CST treatment of NWS-infected MDCK cells did not result in PFU reduction, in contrast, the present results showed that CST-treatment of NWS-N8 infected cells resulted in the reduction of release of infectious virus, along with the reduction of surface expression of the NA molecules. The NWS and the NWS-N8 strains possess the same HA molecule as their surface glycoprotein, on the other hand, the NWS contains the NA of the N1 subtype with 3 potential glycosylation sites, and the NWS-N8 of the N8 subtype with 5 sites. Discrepancy between study by Pan $e t$ al. and ours may reflect the different glucose triming requirement of the NA molecules for their cellular transport. Glucose trimming inhibitors appear to display less anti-viral effect against the virus with the NA having less glycosylation sites. Addition of the exogenous NA did not improve virus particle formation in the presence of 


\section{$\alpha-\mathrm{HA}$}
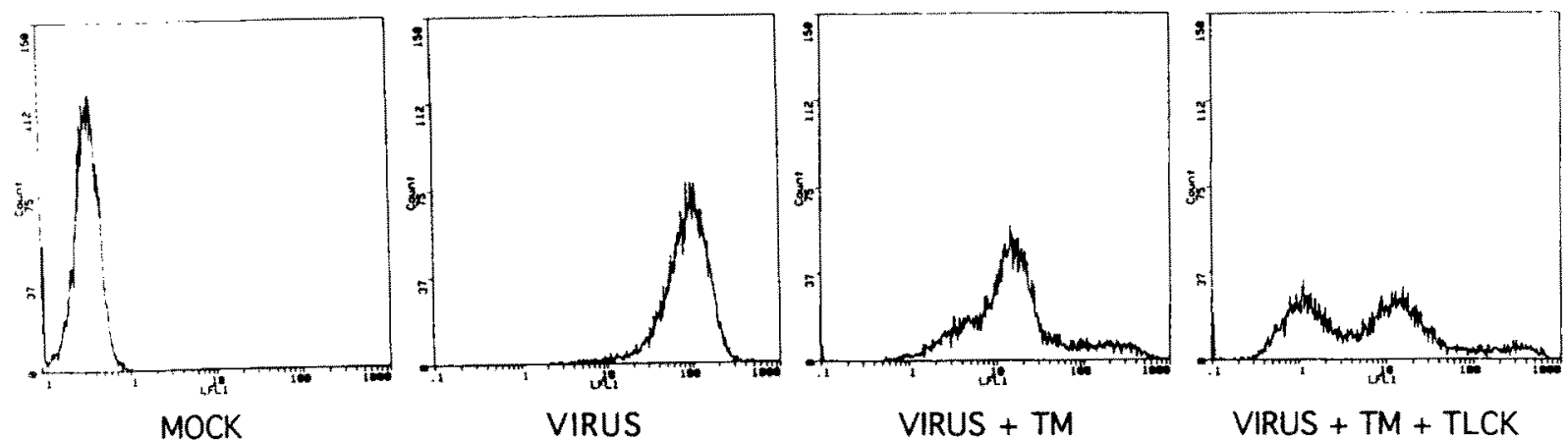

\section{$\alpha-N A(N 8-4)$}
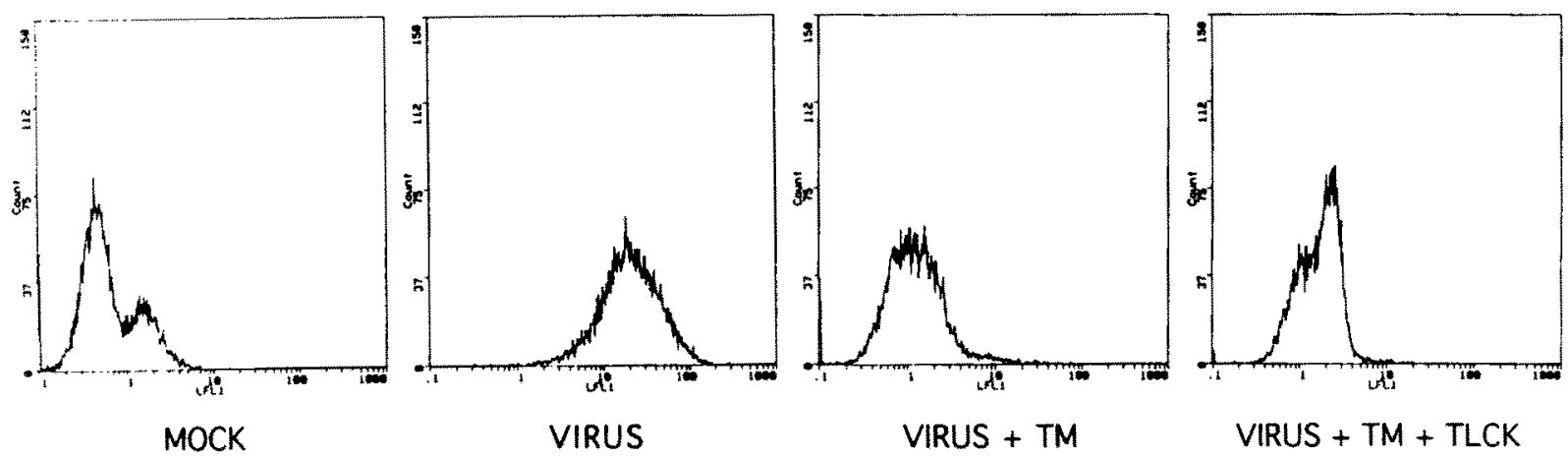

Fig. 5. Effect of CST on the amounts of HA and NA expressed on the surface of the virus-infected cells. Virus-infected cells were treated with CST or CST+TLCK for $5 \mathrm{hr}$ p.i. The cells were then stained with anti-WSN HA MAb (top) and anti-N8 NA MAb N8-4 (bottom).

Table 1. Effect of the exogenous NA on anti-viral activity of CST

\begin{tabular}{ccrr}
\hline & \multicolumn{3}{c}{ Plaque forming unit/m $l^{\mathrm{a})}$} \\
\cline { 2 - 4 } & \multicolumn{3}{c}{ V. cholerae NA added } \\
Virus infection ${ }^{\mathrm{b})}$ & 0 & $5 \mathrm{mU} / \mathrm{m} l$ & $10 \mathrm{mU} / \mathrm{m} l$ \\
\hline NWS-N8 & $9.2 \times 10^{5}$ & $8.8 \times 10^{5}$ & $8.7 \times 10^{5}$ \\
NWS-N8 + CST 1 mM & $1.8 \times 10^{5}$ & $2.0 \times 10^{5}$ & $2.8 \times 10^{5}$ \\
\hline
\end{tabular}

a) Data indicate the mean of duplicate assays for each sample.

b) A confluent monolayer in a 24-well culture plate was infected with NWS-N8 at an m.o.i. of 4. Virus-infected cells were incubated for 17 $\mathrm{hr}$ in the presence or absence of $1 \mathrm{mM} \mathrm{CST}$ as well as the indicated amount of $V$. cholerae NA.

CST, suggesting that the viral NA might be required structurally for viral particle formation. However, Liu and Air [11] reported an NA-deficient virus (NWS-Mvi) with a truncated NA gene. They showed that NA enzymatic activity was not needed for influenza entry, replication, or assembly. Requirement of the NA for the viral particle formation and the replication remains to be elucidated.

In this study, we showed differential requirements of glycosylation and glucose trimming for two viral glycoproteins expressed from a single reassortant influenza A virus strain. The reduced amount of NA surface expression after the treatment with a glucosidase inhibitor suggests that calnexin-like chaperone(s) may play an important role in NA maturation. Whether or not this reduced NA expression at the cell surface accounts for the limited viral particle formation in the presence of CST requires further investigation.

ACKNOWLEDGMENTS. We thank Dr. Robert G. Webster for providing the monoclonal antibodies and NWS-N8 virus, and Dr. Junichi Kawano for his generous help with the FACS analysis.

\section{REFERENCES}

1. Aymard-Henry, M., Colman, M. T., Dowdle, W. R., Laver, W. G., Schild, G. C. and Webster, R .G. 1973. Influenza virus neuraminidase-inhibition test procedures. Bull. W. H. O. 48: 199-202.

2. Chen, W., Helenius, J., Braakman, I. and Helenius, A. 1995. Cotranslational folding and calnexin binding during glycoprotein synthesis. Proc. Natl. Acad. Sci. U.S.A. 92: 62296233.

3. Datema, R., Romero, P. A., Legler, G. and Schwarz, R. T. 1982. Inhibition of formation of complex oligosaccharides by the glucosidase inhibitor bromoconduritol. Proc. Natl. Acad. Sci. U.S.A. 79: 6787-6791. 

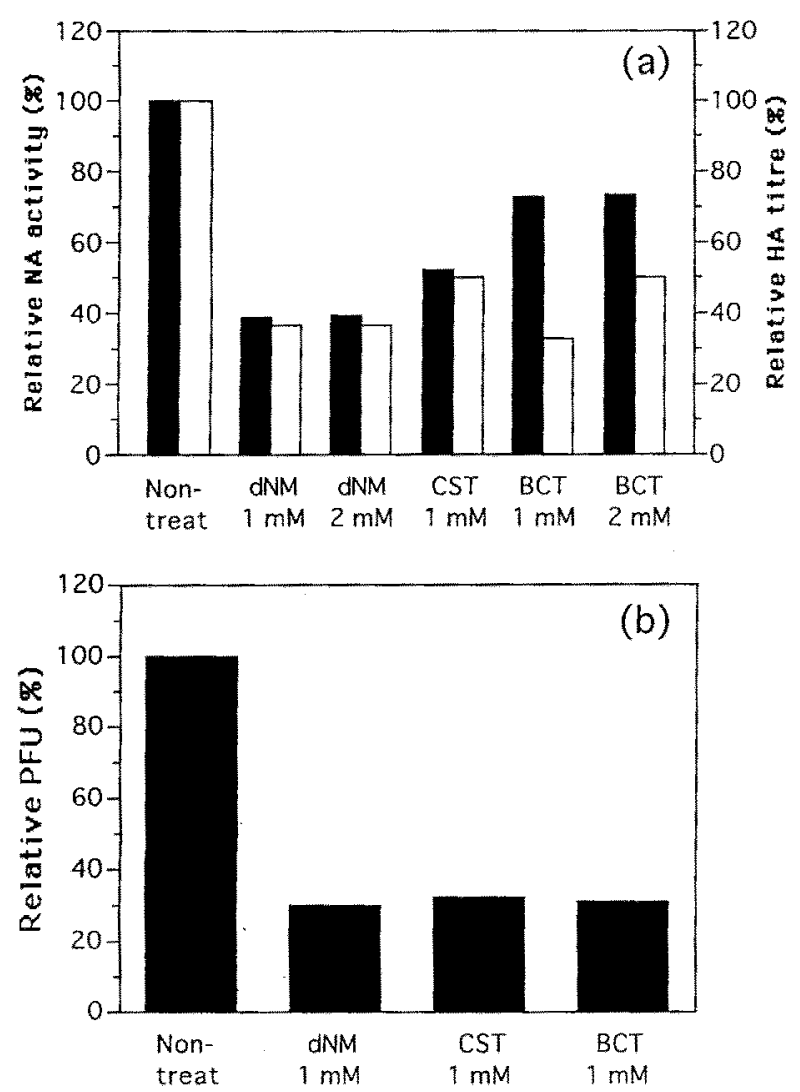

Fig. 6. Inhibition of viral particle formation by glucosidase inhibitors. Culture medium of the virus-infected cells with or without glucosidase inhibitor treatment was harvested $17 \mathrm{hr}$ p.i. NA activity, HA titers and PFU of the culture medium were determined by duplicate assays. Relative NA activity (a; closed column), HA titer (a; open column) and PFU (b) are expressed as percentages of those in the absence of drug treatment.

4. Datema, R., Romero, P. A., Rott, R. and Schwarz, R. T. 1984. On the role of oligosaccharide trimming in the maturation of Sindbis and influenza virus. Arch. Virol. 81: 25-39.

5. Hammond, D., Braakman, I. and Helenius, A. 1994. Role of $\mathrm{N}$-linked oligosaccharide recognition, glucose trimming, and calnexin in glycoprotein folding and quality control. Proc. Natl. Acad. Sci. U.S.A. 91: 913-917.

6. Hammond, C. and Helenius, A. 1994. Folding of VSV G protein: Sequential interaction with Bip and calnexin. Science 266: 456-458.

7. Hebert, D. N., Foellmer, B. and Helenius, A. 1995. Glucose trimming and reglucosylation determine glycoprotein association with calnexin in the endoplasmic reticulum. Cell 81: 425-433.

8. Hebert, D. N., Foellmer, B. and Helenius. A. 1996. Calnexin and calreticulin promote folding, delay oligomerization and suppress degradation of influenza hemagglutinin in microsomes. EMBO J. 15: 2961-2968.

9. Helenius, A. 1994. How N-linked oligosaccharides affect glycoprotein folding in the endoplasmic reticulum. Mol. Biol. Cell 5: 253-265.

10. Klenk, H.-D., Rott, R., Orlich, M. and Blodorn, J. 1975. Activation of influenza A viruses by trypsin treatment. Virology 68: 440-454.

11. Liu, C. and Air, G. M. 1993. Selection and characterization of a neuraminidase minus mutant of influenza virus and its rescue by cloned neuraminidase genes. Virology 194: 403407.

12. McDowell, W., Romero, P. A., Datema, R. and Schwartz, R. T. 1987. Glucose trimming and mannose trimming affect different phases of the maturation of Sindbis virus in infected BHK cells. Virology 161: 37-44.

13. Mitnaul. L. J., Castrucci, M. R., Murti, K. G. and Kawaoka, Y. 1995. The cytoplasmic tail of influenza A virus neuraminidase (NA) incorporation into virions, virion morphology, and virulence in mice but is not essential for virus replication. $J$. Virol. 70: 873-879.

14. Murphy, B.R. and Webster, R. G. 1996. Orthomyxoviruses. pp. 1397-1446. Virology, 3rd ed. (Fields, B. N., Knipe, D. M. and Howley, P. M. eds.), Raven Press Ltd., New York.

15. Ou, W.-J., Cameron, P. H., Thomas, D. Y. and Bergeron, J. M. 1993. Association of folding intermediates of glycoproteins with calnexin during protein maturation. Nature (Lond.) 364: 771-776.

16. Pan, Y. T., Hori, H., Saul, R., Sanford, B. A., Molyneux, R. J. and Elbein, A. D. 1983. Castanospermine inhibits the processing of the oligosaccharide portion of the influenza viral hemagglutinin. Biochemistry 22: 3975-3984.

17. Romero, P. A., Datema, R. and Schwarz, R. T. 1983. Nmethyl-1-deoxynojirimycin, a novel inhibitor of glycoprotein processing, and its effect on fowl plague virus maturation. Virology 130: 238-242.

18. Saito, T., Taylor, G., Laver, W. G., Kawaoka, Y. and Webster, R. G. 1994. Antigenicity of the N8 influenza A virus neuraminidase: Existence of an epitope at the subunit interface of the neuraminidase. J. Virol. 68: 1790-1796.

19. Saito, T., Taylor, G. and Webster, R. G. 1995. Steps in maturation of influenza A virus neuraminidase. J. Virol. 69: 50115017.

20. Smith, M. M., Schlesinger, S., Lindstrom, J. and Merlie, J. P. 1986. The effects of inhibiting oligosaccharide trimming by 1-deoxynojirimycin on the nicotinic acetylcholine receptor. J. Biol. Chem. 261: 14825-14832.

21. Walker, B. D., Kowalski, M., Goh, W. C., Kozarsky, K., Krieger, M., Rosen, C., Rohrschneider, L., Haseltine, W. A. and Sodroski, J. 1987. Inhibition of human immunodeficiency virus syncytium formation and virus replication by castanospermine. Proc. Natl. Acad. Sci. U.S.A. 84: 81208124.

22. Ware, F. E., Vassilakos, A., Peterson, P. A., Jackson, M. R., Lehrman, M. A. and Williams, D. B. 1995. The molecular chaperone calnexin binds Glc1Man9GlcNAc2 oligosaccharide as an initial steps in recognizing unfolded glycoproteins. J. Biol. Chem. 270: 4697-4704. 